\title{
Strongyloides stercoralis infection: an "exotic" cause of chronic diarrhea
}

\author{
Dimitrios S. Politis ${ }^{a}$, Eleni Triantafyllidi ${ }^{b}$, Konstantinos H. Katsanos ${ }^{a}$, Dimitrios K. Christodoulou ${ }^{a}$ \\ University Hospital of Ioannina, Ioannina, Greece
}

A 69-year-old Caucasian male presented with a 6-month history of watery diarrhea without bleeding or weight loss. The patient had received chemotherapy for lung cancer 2 years before the appearance of his symptoms. His laboratory tests revealed eosinophilia, with an absolute eosinophil count of 1950 cells $/ \mu \mathrm{L}$ in peripheral blood, without other inflammatory markers. Stool culture was negative for intestinal pathogens. Computed tomography of the abdomen revealed no abnormal findings. Gastroduodenoscopy and ileocolonoscopy were performed with biopsy samples taken randomly, since the endoscopic findings were normal (Fig. 1). However, the pathology report from duodenal biopsies revealed the presence of chronic inflammatory infiltrate (containing eosinophils), as well as cross-sections of worms with morphological features compatible with Strongyloides stercoralis (S. stercoralis) (Fig. 2).

$S$. stercoralis is an intestinal nematode parasite that has a unique complex life cycle, combining alternating free-living and parasitic reproduction cycles with the capability of autoinfection in the human host. Infection occurs when the filariform larvae from contaminated soil penetrate the host's skin. Its distribution is global, but is considered endemic in developing countries such as those in Southeast Asia and Central America, where sanitation conditions are poor and soil or water sources can be contaminated by feces [1]. Clinical manifestations vary from asymptomatic to diarrhea, bloating or abdominal discomfort, wheezing, cough and urticaria. More severe or lethal manifestations occur with hyperinfection syndrome or disseminated infection due to high parasite load. Stool culture is an insensitive diagnostic method, but agar stool culture plate and serology with ELISA from blood samples lead to higher diagnostic rates [2]. Treatment of choice is ivermectin at a recommended dose of $200 \mu \mathrm{g} / \mathrm{kg} /$ day. Alternative treatments include albendazole and thiabendazole [3].

Departments of a Gastroenterology, University Hospital of Ioannina, Faculty of Medicine, School of Health Sciences, University of Ioannina, Ioannina, Greece (Dimitrios S. Politis, Konstantinos H. Katsanos, Dimitrios K. Christodoulou); bepartment of Pathology, University Hospital of Ioannina, Ioannina, Greece (Eleni Triantafyllidi)

\section{Conflict of Interest: None}

Correspondence to: Dimitrios K. Christodoulou, MD, PhD, Associate Professor of Gastroenterology, Department of Gastroenterology, University Hospital of Ioannina, Faculty of Medicine, School of Health Sciences, University of Ioannina, 45110 Ioannina, Greece, Tel.: +30 26510 07501, Fax: +30 2651007016 ,

e-mail: dchristodoulou@gmail.com

Received 12 April 2017; accepted 25 April 2017; published online 12 May 2017

DOI: https://doi.org/10.20524/aog.2017.0160

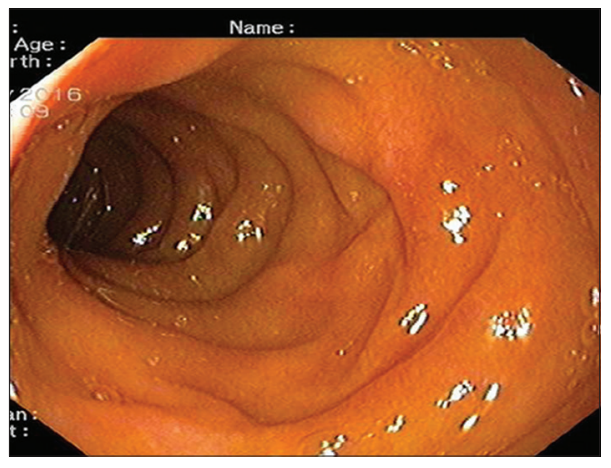

Figure 1 Normal endoscopic appearance of the second part of the duodenum

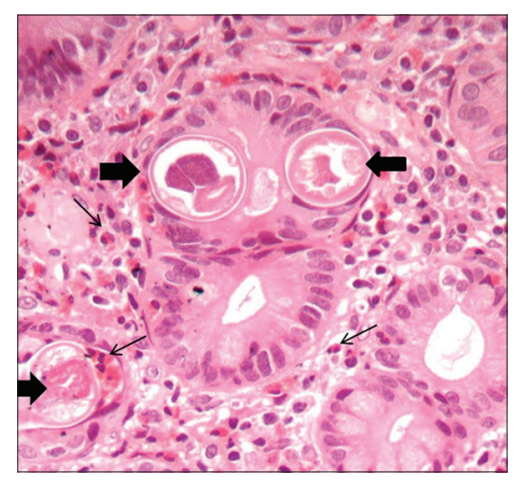

Figure 2 Small intestinal biopsy with evident worms lying within crypts and prominent eosinophilic infiltration (thin black arrows). Transverse cross-section shows the characteristic intestine and pair of reproductive tubes (thick black arrows). Hematoxylin\&eosin $\times 400$

\section{Acknowledgment}

The authors would like to thank Dr. Anna Batistatou, Professor of Pathology, for her valuable contribution and comments on the pathology report.

\section{References}

1. Puthiyakunnon S, Boddu S, Li Y, et al. Strongyloidiasis—an insight into its global prevalence and management. PLoS Negl Trop Dis 2014;8:e3018.

2. Segarra-Newnham M. Manifestations, diagnosis, and treatment of Strongyloides stercoralis infection. Ann Pharmacother 2007;41:1992-2001.

3. Luvira V, Watthanakulpanich D, Pittisuttithum P. Management of Strongyloides stercoralis: a puzzling parasite. Int Health 2014;6:273-281. 